
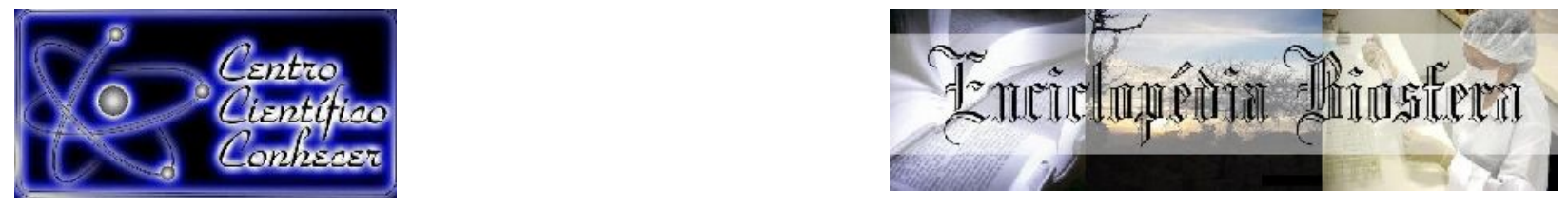

\title{
EFEITOS DA EQUOTERAPIA SOBRE A ANSIEDADE E QUALIDADE DE VIDA DE UM IDOSO INSTITUCIONALIZADO: UM RELATO DE CASO
}

Ana Clara Aguilar Santos ${ }^{1}$, Amanda Melo Sant'anna Araújo², Débora Ribeiro Orlando $^{3}$, Adalfredo Rocha Lobo-Júnior ${ }^{4}$, Eric Francelino Andrade ${ }^{5}$

1 Graduanda em Medicina Veterinária, Universidade Federal dos Vales do Jequitinhonha e Mucuri (UFVJM), Unaí, MG - Brasil.

2 Profa. Mestre, Universidade Federal dos Vales do Jequitinhonha e Mucuri (UFVJM), Unaí, $M G$ - Brasil.

3 Profa. Doutora, Universidade Federal dos Vales do Jequitinhonha e Mucuri (UFVJM), Unaí, MG - Brasil.

4 Prof. Doutor, Universidade Federal dos Vales do Jequitinhonha e Mucuri (UFVJM), Unaí, MG - Brasil

5 Prof. Doutor, Universidade Federal dos Vales do Jequitinhonha e Mucuri (UFVJM), Unaí, MG - Brasil (ericfrancelinoandrade@gmail.com)

Recebido em: 04/10/2019 - Aprovado em: 30/11/2019 - Publicado em: 15/12/2019 DOI: 10.18677/EnciBio_2019B24

\section{RESUMO}

A equoterapia é uma terapia assistida por animais que promove diversos benefícios para a saúde física e mental. A literatura é escassa no que diz respeito aos efeitos da prática de equoterapia sobre a qualidade de vida e ansiedade de idosos institucionalizados. Desta forma, o presente relato de caso objetivou avaliar os efeitos da prática de equoterapia sobre a qualidade de vida e ansiedade de um idoso. O paciente, saudável, com 85 anos de idade, residente em uma casa de repouso no noroeste do Estado de Minas Gerais, praticou equoterapia por sete semanas. Cada sessão da terapia consistiu em 30 minutos de atividades para promoção de equilíbrio, postura e autoconfiança. Antes da primeira, e ao final da última sessão de equoterapia o idoso respondeu ao Inventário de Ansiedade TraçoEstado (IDATE) e ao "World Health Organization Quality of Life- Bref" (WHOQOLBref). Os dados foram submetidos à Análise de Variância e ao teste do qui-quadrado e exato de Fisher $(p<0,05)$. Observou-se que após o período de equoterapia os níveis de ansiedade-estado diminuíram significativamente. Os escores de qualidade de vida geral e do domínio meio ambiente aumentaram de forma significativa após as sete semanas de equoterapia. Conclui-se que a prática de equoterapia melhorou os escores de ansiedade e a qualidade de vida do idoso avaliado.

PALAVRAS-CHAVE: Ansiedade. Atividade Assistida por Animais. Hipoterapia.

\section{EFFECTS OF EQUINE-ASSISTED THERAPY ON ANXIETY AND QUALITY OF LIFE OF AN INSTITUTIONALIZED ELDERLY: A CASE REPORT}

\section{ABSTRACT}

Equine-assisted therapy is an animal-assisted therapy that promotes many benefits for physical and mental health. Literature is scarce regarding the effects of the 
hippotherapy practice on the quality of life and anxiety of institutionalized elderly. Thus, this case report aimed to evaluate the effects of hippotherapy practice on the quality of life and anxiety of an elderly. The healthy patient with 85-year-old living in a nursing home in the northwest of Minas Gerais practiced equine-assisted therapy for seven weeks. Each therapy session consisted of 30 minutes of activities to stimulates balance, posture and self-confidence. Prior to the first, and at the end of the last equine-assisted therapy session, the elderly responded to the State-Trait Anxiety Inventory (STAI) and the World Health Organization Quality of Life-Bref (WHOQOLBref). Data were submitted to analysis of variance, chi-square test and Fisher's exact test $(p<0,05)$. We observed that after the equine-assisted therapy period the stateanxiety levels decreased significantly. Overall quality of life and environmental domain scores increased significantly after seven weeks of hippotherapy. We concluded that the equine-assisted therapy practice improved both scores of anxiety and quality of life of the evaluated elderly.

PALAVRAS-CHAVE: Anxiety. Animal Assisted Activity. Hippotherapy.

\section{INTRODUÇÃO}

A qualidade de vida é considerada como a percepção do indivíduo sobre sua posição na vida em um contexto cultural e de sistema de valores em que vive, bem como sua relação com metas, expectativas, padrões e preocupações (THE WHOQUOL GROUP, 1998a). Este termo está intimamente relacionado a fatores como status socioeconômico, percepção de bem-estar e aspectos relacionados à saúde física e mental (KARIMI; BRAZIER, 2016). Pacientes como baixos escores de qualidade de vida geralmente podem apresentar sintomas de depressão e ansiedade (LI et al., 2016). A ansiedade é considerada um distúrbio caracterizado pela antecipação a uma ameaça iminente que, pode ou não existir, e que promove sentimentos de incerteza, pavor e medo (STEIN; SAREEN, 2015).

Esta condição é uma importante resposta adaptativa para manutenção da vida, que prepara o organismo para eventos adversos e outras ameaças (COHEN et al., 2016). Entretanto, quando excessiva ou persistente, a ansiedade pode ser o gatilho para desencadear mecanismos relacionados à diversas doenças (NILES et al., 2015; OUAKININ, 2016). Comumente, pacientes com transtornos de ansiedade podem apresentar desordem de ansiedade generalizada, transtorno do pânico, transtorno de ansiedade social e transtorno de estresse pós-traumático (STEIN; CRASKE, 2017).

As abordagens farmacológicas atuais envolvem ansiolíticos de efeito rápido (benzodiazepínicos e alguns antipsicóticos) ou o tratamento crônico/prolongado (antidepressivos) para atenuar os sintomas de ansiedade (SINGEWALD et al., 2015). Todavia, $40 \%$ dos pacientes apresentam apenas melhorias parciais a longo prazo, e, a maioria deles falha em alcançar remissão completa (SINGEWALD et al., 2015). Nesse sentido, alternativas complementares têm sido associadas ao tratamento farmacológico para melhorar os resultados dos pacientes (STEIN; CRASKE, 2017). Dentre as alternativas não-farmacológicas para o tratamento da ansiedade, destaca-se a terapia assistida por animais (TAA) (WAITE et al., 2018).

A TAA é definida como qualquer intervenção que incorpora o animal como componente do tratamento (O'HAIRE et al., 2015). Os tipos de TAA se estendem desde o uso de pequenos animais como peixes e hamsters até cavalos (RAJE et al., 2018). A equoterapia ou hipoterapia é a TAA mais comumente usada para o tratamento de problemas neuromotores ou deficiências físicas (STERGIOU et al., 
2017). Adicionalmente, os efeitos da equoterapia sobre condições psicológicas como estresse e depressão têm sido investigadas (ALFONSO et al., 2015; OUAKININ, 2016; JOHNSON et al., 2018). Entretanto, poucos estudos têm avaliado os efeitos da equoterapia especificamente sobre a ansiedade e qualidade de vida do paciente residente em instituições ou abrigos. Idosos institucionalizados comumente podem apresentar piora na qualidade de vida, além de aumento nos níveis de ansiedade (RASQUINHA; ACHARYA, 2013; AMONKAR et al., 2018). Assim, o presente relato de caso objetivou avaliar os efeitos de sete semanas de prática de equoterapia sobre a qualidade de vida e ansiedade de um idoso institucionalizado.

\section{RELATO DE CASO}

O presente relato de caso foi desenvolvido no espaço destinado ao projeto de extensão "Equoterapia: um olhar sobre quatro patas", realizado nas dependências da Universidade Federal dos Vales do Jequitinhonha e Mucuri (UFVJM) - Campus Unaí. Antes da coleta de dados, o voluntário assinou o Termo de Consentimento Livre (TCL) que garantia a confidencialidade, bem como deixava claro que, em qualquer momento, o participante poderia abandonar o estudo.

O voluntário do sexo masculino (85 anos de idade) residente, desde 06/11/2012, no Abrigo Frei Anselmo da Sociedade São Vicente de Paula -SSVP da cidade de Unaí - MG, apresentava cognitivo preservado e foi atestado pelo Médico da instituição como apto para a prática das atividades de equoterapia. As queixas principais do voluntário eram a deficiência parcial auditiva e visual em um dos ouvidos e olhos, respectivamente. A seleção do indivíduo para o presente relato de caso deu-se devido ao fato de que, dentre os idosos participantes do projeto, este foi o único paciente que realizou todas as sessões de equoterapia no intervalo de sete semanas.

\section{Instrumentos}

Antes da primeira sessão de equoterapia o idoso respondeu, em forma de entrevista, ao "World Health Organization Quality of Life-Bref' (WHOQOL-Bref) e ao Inventário de Ansiedade Traço-Estado (IDATE).

O WHOQOL-Bref é um instrumento desenvolvido pela Organização Mundial da Saúde (THE WHOQOL GROUP, 1998b) e contém 26 questões com respostas em escala Likert que permitem a avaliação e quantificação do nível da qualidade de vida do paciente. O escore de qualidade de vida é obtido de acordo com o somatório das respostas apresentadas, onde, quanto maior o valor, melhor a qualidade de vida. Desta forma, das 26 questões, duas estão relacionadas à qualidade de vida geral, e os 24 itens restantes são distribuídos nos domínios físico, psicológico, relações sociais e meio ambiente. A classificação da qualidade de vida é então obtida de forma geral e para cada domínio específico.

O IDATE, é um instrumento que permite a avaliação da ansiedade em seus domínios ansiedade-traço (AT) e ansiedade-estado (AE) (SPIELBERGER et al., 1970 ) e possui 40 questões (20 relacionadas à $A T$ e outras 20 à $A E$ ). Cada questão deste instrumento permite respostas em escala Likert que variam de: "muitíssimo", "bastante", "um pouco", e "absolutamente não". A classificação do nível de ansiedade é obtida pelo somatório dos valores apresentados nas questões para ambos os domínios (LORICCHIO; LEITE, 2012). Assim, quanto maior o valor atribuído, maior nível de ansiedade (LORICCHIO; LEITE, 2001; ANDRADE et al., 2018). 
Após a última sessão de equoterapia, os mesmos instrumentos foram aplicados novamente. As coletas de dados foram realizadas pelo mesmo pesquisador no ambiente em que ocorreram as sessões de equoterapia. $\mathrm{O}$ tempo de aplicação foi livre, de acordo com a necessidade do voluntário.

\section{Procedimentos}

Após a entrevista inicial quando foram aplicados os instrumentos mencionados, o voluntário foi encaminhado para a primeira sessão de equoterapia. A primeira estratégia realizada foi a explicação, de forma terapêutica, sobre a equoterapia ao paciente. Foi abordado sobre a importância de realizar uma atividade que envolve a participação do idoso e os potenciais benefícios da prática. Ao mesmo tempo era permitido ao paciente afagar e escovar o cavalo. As sete sessões de equoterapia foram realizadas com duração em média de 30 minutos cada, tendo início em setembro de 2018 e término em novembro do mesmo ano.

$\mathrm{O}$ animal utilizado tinha, aproximadamente, um metro e setenta centímetros de altura de cernelha, era adestrado e manso. Durante a trajetória, o paciente montava no cavalo com auxílio da equipe e era conduzido pelo fisioterapeuta especializado e por mais dois alunos voluntários, de forma que um apoiava lateralmente o paciente e o outro conduzia o cavalo ditando o ritmo da passada.

Durante 0 atendimento, eram realizadas cinco voltas sobre o percurso para adaptação do praticante com 0 animal e 0 ambiente. Em seguida 0 paciente realizava atividades de equilíbrio, cooperação, concentração e alongamento nos membros superiores e tronco, conforme aceitação e preferência pessoal do participante.

Em momentos específicos das sessões eram empregados materiais variados como bambolê, boliche, argola e circuitos com obstáculos em que o cavalo percorria em ziguezague, com intuito de promover estímulos para o equilíbrio e ganho que amplitude de movimento. Adicionalmente, eram realizadas paradas no percurso em frente de um espelho para que o paciente corrigisse a postura e se visualizasse sobre o cavalo de forma a promover estímulos para a autoconfiança e autoestima.

\section{Análises estatísticas}

Para comparar as porcentagens de frequência (proporções) e do número de vezes que um item foi marcado dentro da série de questões relacionadas à ansiedade e qualidade de vida no paciente antes e depois das sessões de equoterapia, foi aplicado o teste do qui-quadrado e teste exato de Fisher (células com contagem esperada menor que 5). Além disso, a análise de variância (teste $F$ ) também foi realizada para comparar as médias dos escores para cada série de questões, antes e depois do período de equoterapia. Foram consideradas diferenças significativas um nível de probabilidade menor que $5 \%(p<0,05)$. Finalmente, as comparações relativas de frequência e médias foram, respectivamente, realizadas utilizando o FREQ e MIXED do pacote estatístico do software Statistical Analysis System (SAS Inst. Inc., Cary, NC, USA; versão 9.2).

\section{RESULTADOS E DISCUSSÃO}

Os escores de ansiedade-estado diminuíram significativamente $(p<0,05)$ após as sete semanas de equoterapia (Tabelas 1 e 2). Entretanto, não foram observadas diferenças entre os escores de ansiedade-traço. Com relação à qualidade de vida, foi observado aumento significativo $(p<0,05)$ na qualidade de 
vida geral e no domínio meio ambiente (Tabelas 1 e 2). Não houveram diferenças nos domínios físico, psicológicos e relações sociais após o período de equoterapia.

TABELA 1 - Porcentagens de frequência (proporções) do número de vezes que um item foi marcado dentro da série de questões relacionadas à ansiedade-estado e ansiedade-traço no IDADE ou relacionadas aos domínios de qualidade de vida no WHOQUOL-Bref de um paciente idoso antes e depois de sete semanas de equoterapia.

\begin{tabular}{|c|c|c|c|c|c|}
\hline \multirow[b]{2}{*}{ Período } & \multicolumn{5}{|c|}{ Item marcado no IDATE } \\
\hline & \multicolumn{2}{|c|}{$\begin{array}{c}1 \\
\begin{array}{c}\text { (absolutamente } \\
\text { não) }\end{array}\end{array}$} & $\begin{array}{c}2 \\
\text { (um } \\
\text { pouco) }\end{array}$ & $\begin{array}{c}3 \\
\text { (bastante) }\end{array}$ & $\begin{array}{c}4 \\
\text { (muitíssimo) }\end{array}$ \\
\hline \multicolumn{6}{|c|}{ Ansiedade-Estado } \\
\hline Antes $(n q=20)$ & \multicolumn{2}{|c|}{$55.0(11)$} & $5.0(1)$ & $20.0(4)$ & $20.0(4)$ \\
\hline Depois $(n q=20)$ & \multicolumn{2}{|c|}{$75.0(15)$} & $25.0(5)$ & $0.0(0)$ & $0.0(0)$ \\
\hline Valor de $\mathrm{p}$ (qui-quadrado) & \multicolumn{2}{|c|}{0.1848} & 0.0765 & 0.0350 & 0.0350 \\
\hline Valor de $\mathrm{p}$ (Fisher) & \multicolumn{2}{|c|}{0.3203} & 0.1818 & 0.1060 & 0.1060 \\
\hline \multicolumn{6}{|c|}{ Ansiedade-Traço } \\
\hline Antes $(n q=20)$ & \multicolumn{2}{|c|}{$30.0(6)$} & $10.0(2)$ & $10.0(2)$ & $50.0(10)$ \\
\hline Depois $(n q=20)$ & \multicolumn{2}{|c|}{$25.0(5)$} & $40.0(8)$ & $25.0(5)$ & $10.0(2)$ \\
\hline Valor de $\mathrm{p}$ (qui-quadrado) & \multicolumn{2}{|c|}{0.7233} & 0.0285 & 0.2119 & 0.0058 \\
\hline Valor de $\mathrm{p}$ (Fisher) & \multicolumn{2}{|c|}{1.0000} & 0.0648 & 0.4075 & 0.0138 \\
\hline \multirow[b]{2}{*}{ Período } & \multicolumn{5}{|c|}{ Item marcado no WHOQUOL-Bref } \\
\hline & $\begin{array}{c}1 \\
\text { (nada) }\end{array}$ & $\begin{array}{c}2 \\
\text { (muito } \\
\text { pouco) }\end{array}$ & $\begin{array}{c}3 \\
\text { (médio) }\end{array}$ & $\begin{array}{c}4 \\
\text { (muito) }\end{array}$ & $\begin{array}{c}5 \\
\begin{array}{c}\text { (completamen } \\
\text { te) }\end{array}\end{array}$ \\
\hline \multicolumn{6}{|c|}{ Qualidade de Vida Geral } \\
\hline Antes $(n q=20)$ & $\begin{array}{l}42.3 \\
(11)\end{array}$ & $19.2(5)$ & $15.4(4)$ & $0.0(0)$ & $23.1(6)$ \\
\hline Depois $(n q=20)$ & $19.2(5)$ & $11.5(3)$ & $15.4(4)$ & $23.1(6)$ & $30.8(8)$ \\
\hline Valor de $\mathrm{p}$ (qui-quadrado) & 0.0714 & 0.4421 & 1.0000 & 0.0092 & 0.5318 \\
\hline Valor de $\mathrm{p}$ (Fisher) & 0.1318 & 0.7030 & 1.0000 & 0.0226 & 0.7554 \\
\hline \multicolumn{6}{|c|}{ Domínio Físico } \\
\hline Antes $(n q=20)$ & $42.9(3)$ & $0.0(0)$ & $14.3(1)$ & $0.0(0)$ & $42.9(3)$ \\
\hline Depois $(n q=20)$ & $42.9(3)$ & $14.3(1)$ & $0.0(0)^{\prime}$ & $28.6(2)$ & $14.3(1)$ \\
\hline Valor de $\mathrm{p}$ (qui-quadrado) & 1.0000 & 0.2994 & 0.2994 & 0.1266 & 0.2367 \\
\hline Valor de $\mathrm{p}$ (Fisher) & 1.0000 & 1.0000 & 1.0000 & 0.4615 & 0.5594 \\
\hline \multicolumn{6}{|c|}{ Domínio Psicológico } \\
\hline Antes $(n q=20)$ & $50.0(3)$ & $0.0(0)$ & $16.7(1)$ & $0.0(0)$ & $33.3(2)$ \\
\hline Depois $(n q=20)$ & $16.7(1)$ & $33.3(2)$ & $33.3(2)$ & $16.7(1)$ & $0.0(0)$ \\
\hline Valor de $\mathrm{p}$ (qui-quadrado) & 0.2207 & 0.1213 & 0.5050 & 0.2963 & 0.1213 \\
\hline Valor de $\mathrm{p}$ (Fisher) & 0.5455 & 0.4545 & 1.0000 & 1.0000 & 0.4545 \\
\hline \multicolumn{6}{|c|}{ Domínio Relações Sociais } \\
\hline Antes $(n q=20)$ & $33.3(1)$ & $66.7(2)$ & $0.0(0)$ & $0.0(0)$ & $0.0(0)$ \\
\hline Depois $(n q=20)$ & $33.3(1)$ & $0.0(0)$ & $0.0(0)$ & $33.3(1)$ & $33.3(1)$ \\
\hline Valor de $\mathrm{p}$ (qui-quadrado) & 1.0000 & 0.0833 & - & 0.2733 & 0.2733 \\
\hline Valor de $\mathrm{p}$ (Fisher) & 1.0000 & 0.4000 & - & 1.0000 & 1.0000 \\
\hline \multicolumn{6}{|c|}{ Domínio Meio Ambiente } \\
\hline Antes $(n q=20)$ & $25.0(2)$ & $37.5(3)$ & $25.0(2)$ & $0.0(0)$ & $12.5(1)$ \\
\hline Depois $(n q=20)$ & $0.0(0)$ & $0.0(0)$ & $0.0(0)$ & $25.0(2)$ & $75.0(6)$ \\
\hline Valor de $\mathrm{p}$ (qui-quadrado) & 0.1306 & 0.0547 & 0.1306 & 0.1306 & 0.0117 \\
\hline Valor de $\mathrm{p}$ (Fisher) & 0.4667 & 0.2000 & 0.4667 & 0.4667 & 0.0406 \\
\hline
\end{tabular}


Porcentagem de frequência (número de vezes que uma resposta foi marcada); $n q$ = número de questões. Atenção: Nos casos de células com contagem esperada menor que 5, o teste do qui-quadrado pode não ser válido. Então, o teste exato de Fisher deve ser usado.

TABELA 2 - Escores médios para cada domínio relacionado à ansiedade e qualidade de vida de um idoso institucionalizado antes e depois de sete semanas de equoterapia.

\begin{tabular}{|c|c|c|c|c|}
\hline \multirow{2}{*}{ Domínio comportamental } & \multicolumn{2}{|c|}{ Período } & \multirow{2}{*}{ EP } & \multirow{2}{*}{ Valor de $p$ (teste $F$ ) } \\
\hline & Antes & Depois & & \\
\hline Ansiedade-Estado & 2.1 & $1.3^{*}$ & 0.21 & 0.0117 \\
\hline Ansiedade-Traço & 2.8 & 2.2 & 0.26 & 0.1144 \\
\hline Qualidade de Vida Geral & 2.4 & $3.3^{*}$ & 0.31 & 0.0382 \\
\hline Domínio Físico & 3.0 & 2.6 & 0.70 & 0.6748 \\
\hline Domínio Psicológico & 2.7 & 2.5 & 0.64 & 0.8583 \\
\hline Domínio Relações Sociais & 1.7 & 3.3 & 0.88 & 0.2524 \\
\hline Domínio Meio Ambiente & 2.4 & $4.8^{*}$ & 0.35 & 0.0003 \\
\hline
\end{tabular}

$\mathrm{EP}=$ Erro padrão. ${ }^{*}$ Diferença significativa $(\mathrm{p}<0,05)$.

Os benefícios psicológicos, comportamentais e motores frente à prática de equoterapia são amplamente descritos na literatura (MARTíN-VALERO et al., 2018; PEPPE et al., 2018; ROMANIUK et al., 2018; MENDONÇA et al., 2019). Entretanto, são escassos os estudos avaliando, simultaneamente, o efeito desta TAA sobre a ansiedade e a qualidade de vida, principalmente quando se trata de idosos institucionalizados. A diminuição da ansiedade-estado observada no idoso do presente relato de caso é justificada devido ao fato de este componente estar relacionado a um estado situacional e transitório no indivíduo (DIBBETS; EVERS, 2017).

'Desta forma, o contato com o animal pode desencadear respostas orgânicas que diminuem o estado de alerta e apreensão constantes em um indivíduo ansioso (NEPPS et al., 2014). Foi observado em estudo prévio que a prática de equoterapia durante seis semanas (uma sessão semanal com duração de duas horas) promoveu diminuição nos sintomas de ansiedade em pacientes com síndrome de estresse póstraumático (EARLES et al., 2015). No presente relato, não foi observada diminuição significativa na ansiedade-traço do paciente, uma vez que esta é menos passível de modificação, já que é uma característica da personalidade do indivíduo (SPIELBERGER et al., 1970). Apenas sete semanas de terapia não seriam suficientes para promover esta alteração, como observado no idoso avaliado.

Os benefícios da equoterapia sobre a qualidade foram evidenciados em uma recente umbrela review, que é uma revisão de revisões de literatura (STERN; CHUR-HANSEN, 2019). O fato do domínio meio ambiente ter sido o único a apresentar aumento significativo dentre os demais é interessante devido ao idoso avaliado ser institucionalizado. Como cada domínio da qualidade de vida é multifatorial, possivelmente, o contato e permanência em um ambiente externo ao da instituição em que reside tenha sido um fator de melhoria para este domínio da qualidade de vida. Além disso o fato do ambiente onde ocorreu a equoterapia ser arborizado e calmo, também pode ser estímulo de melhoria deste domínio uma vez que este considera aspectos como clima, barulho e poluição (SOUZA et al., 2018). 
Surpreendentemente, o idoso avaliado não apresentou aumento significativo no domínio físico após o período de equoterapia. Um dos principais objetivos desta TAA é a melhora de fatores como equilíbrio e coordenação (HILLIERE et al., 2018). Tais fatores, estão diretamente relacionados ao domínio físico da qualidade de vida (ROBERTS-CLARKE et al., 2016). Adicionalmente, considerou-se que apenas sete sessões de equoterapia não tenham sido suficientes para melhoria dos domínios físico, psicológico e relações sociais, uma vez que estes necessitem de intensidade e frequência maior de estímulos para serem alterados significativamente.

A prática de equoterapia em idosos é bastante relatada na literatura devido aos benefícios como melhora do equilíbrio, aumento da autoestima e autoconfiança (ARAUJO et al., 2011; DUFFY, 2018). O presente relato de caso foi realizado com apenas um idoso devido ao fato de este ter sido o único que manteve aderência às sessões durante o período de sete semanas. Os resultados do presente relato encorajam prática de equoterapia em idosos institucionalizados. Além disso, é interessante que a ansiedade e qualidade de vida sejam avaliados em grupos maiores de idosos e em períodos maiores de tempo.

\section{CONCLUSÃO}

A prática de equoterapia por sete semanas melhorou os níveis de ansiedadeestado, qualidade de vida geral e o domínio meio ambiente da qualidade de vida de um idoso institucionalizado.

\section{AGRADECIMENTOS}

Os autores agradecem ao Conselho Nacional de Desenvolvimento Científico e Tecnológico ( $\mathrm{CNPq}$ ) e à Universidade Federal dos Vales do Jequitinhonha e Mucuri, pelo apoio fornecido. Em especial, agradecemos ao fisioterapeuta Wesley Alves.

\section{REFERÊNCIAS}

ALFONSO, S. V.; ALFONSO, L. A.; LLABRE, M. M.; FERNANDEZ, M. I. Project Stride: An Equine-Assisted Intervention to Reduce Symptoms of Social Anxiety in Young Women. EXPLORE, v. 11, n. 6, p. 461-467, 2015. Disponível em: < https://www.ncbi.nlm.nih.gov/pubmed/26386749>. 10.1016/j.explore.2015.08.003.

AMONKAR, P.; MANKAR, M. J.; THATKAR, P.; SAWARDEKAR, P.; GOEL, R.; et al. A comparative study of health status and quality of life of elderly people living in old age homes and within family setup in Raigad District, Maharashtra. Indian Journal of Community Medicine, v. 43, n. 1, p. 10, 2018. Disponível em: < https://www.ncbi.nlm.nih.gov/pubmed/29531431 >. doi: 10.4103/ijcm.IJCM_301_16.

ANDRADE, K. A.; ARAÚJO, A. M. S.; ANDRADE, E. F.; ORLANDO, D. R. Universitários que convivem com animais de estimação apresentam menores níveis de ansiedade. Enciclopédia Biosfera, v. 15, n. 28, p. 896-906, 2018. Disponível em: < http://www.conhecer.org.br/enciclop/2018B/AGRAR/universitarios.pdf>. doi: 10.18677/EnciBio_2018B73

ARAUJO, T. B.; SILVA, N. A.; COSTA, J. N.; PEREIRA, M. M.; SAFONS, M. P. Effect of equine-assisted therapy on the postural balance of the elderly. Brazilian 
Journal of Physical Therapy, v. 15, n. 5, p. 414-419, 2011. Disponível em: < http://www.scielo.br/scielo.php?script=sci_arttext\&pid=S1413-35552011000500012>. doi: 10.1590/S1413-35552011005000027.

COHEN, S. D.; CUKOR, D.; KIMMEL, P. L. Anxiety in Patients Treated with Hemodialysis. Clinical Journal of the American Society of Nephrology, v. 11, n. 12, p. 2250-2255, 2016. Disponível em: < https://cjasn.asnjournals.org/content/11/12/2250.long >. doi: 10.2215/CJN.02590316

DIBBETS, P.; EVERS, E. A. T. The Influence of State Anxiety on Fear Discrimination and Extinction in Females. Frontiers in Psychology, v. 8, n. 347, p. 1-12, 2017. Disponível em: < https://www.ncbi.nlm.nih.gov/pmc/articles/PMC5352667/>. doi: 10.3389/fpsyg.2017.00347

DUFFY, A.-M. Equine-Assisted Learning in Mental Health Care: A Natural Fit with Recreation Therapy? Literature Review Equine-Assisted Learning in Mental Health Care: A Natural Fit with Recreation Therapy? Journal of Therapeutic Recreation Ontario, v. 13, p. 87-98, 2018. Disponível em: < http://www.horsemuse.ca/uploads/1/1/0/8/110818405/equine_assisted_learning_men tal_health_care.pdf>.

EARLES, J. L.; VERNON, L. L.; YETZ, J. P. Equine-Assisted Therapy for Anxiety and Posttraumatic Stress Symptoms. Journal of Traumatic Stress, v. 28, n. 2, p. 149152, 2015. Disponível em: <https://www.ncbi.nlm.nih.gov/pubmed/25782709>. doi: $10.1002 /$ jts.21990

HILLIERE, C.; COLLADO-MATEO, D.; VILLAFAINA, S.; DUQUE-FONSECA, P.; PARRAÇA, J. A. Benefits of Hippotherapy and Horse Riding Simulation Exercise on Healthy Older Adults: A Systematic Review. PM \& R: The Journal of Injury, Function and Rehabilitation Homepage, v. 10, n. 10, p. 1062-1072, 2018. Disponível em: <https://onlinelibrary.wiley.com/doi/abs/10.1016/j.pmrj.2018.03.019>. doi: 10.1016/j.pmrj.2018.03.019

JOHNSON, R. A.; ALBRIGHT, D. L.; MARZOLF, J. R.; BIBBO, J. L.; YAGLOM, H. D.; et al. Effects of therapeutic horseback riding on post-traumatic stress disorder in military veterans. Military Medical Research, v. 5, n. 1, p. 3, 19-32, 2018. Disponível em: < https://mmrjournal.biomedcentral.com/articles/10.1186/s40779-0180149-6>. doi: 10.1186/s40779-018-0149-6.

KARIMI, M.; BRAZIER, J. Health, Health-Related Quality of Life, and Quality of Life: What is the Difference? PharmacoEconomics, v. 34, n. 7, p. 645-649, 2016. Disponível em: < https://www.ncbi.nlm.nih.gov/pubmed/26892973>. doi: $10.1007 / \mathrm{s} 40273-016-0389-9$

LI, Y.-N.; SHAPIRO, B.; KIM, J. C.; ZHANG, M.; PORSZASZ, J.; et al. Association between quality of life and anxiety, depression, physical activity and physical performance in maintenance hemodialysis patients. Chronic Diseases and Translational Medicine, v. 2, n. 2, p. 110-119, 2016. Disponível em: <https://www.ncbi.nlm.nih.gov/pubmed/29063031>. doi: 10.1016/j.cdtm.2016.09.004. 
LORICCHIO, T. M. B.; LEITE, J. R. Estresse, ansiedade, crenças de autoeficácia e o desempenho dos bacharéis em Direito. Avaliação Psicológica, v. 11, n. 1, p. 3747, $2012 . \quad$ Disponível em: <http://pepsic.bvsalud.org/scielo.php?script=sci_arttext\&pid=S167704712012000100005>.

MARTÍN-VALERO, R.; VEGA-BALLÓN, J.; PEREZ-CABEZAS, V. Benefits of hippotherapy in children with cerebral palsy: A narrative review. European Journal of Paediatric Neurology, v. 22, n. 6, p. 1150-1160, 2018. Disponível em: <https://www.ncbi.nlm.nih.gov/pubmed/30017618>. doi: 10.1016/j.ejpn.2018.07.002

MENDONÇA, T., BIENBOIRE-FROSINI, C.; MENUGE, F.; LECLERCQ, J.; LAFONTLECUELLE, C.; et al. The Impact of Equine-Assisted Therapy on Equine Behavioral and Physiological Responses. Animals, v. 9, n. 7, p. 409-421, 2019. Disponível em: < https://www.mdpi.com/2076-2615/9/7/409/htm>. doi: 10.3390/ani9070409

NEPPS, P.; STEWART, C. N.; BRUCKNO, S. R. Animal-Assisted Activity: Effects of a Complementary Intervention Program on Psychological and Physiological Variables. Journal of Evidence-Based Complementary \& Alternative Medicine, v. 19, n. 3 , p. 211-215, 2014. Disponível em: < https://www.ncbi.nlm.nih.gov/pubmed/24789913>. doi: 10.1177/2156587214533570.

NILES, A. N.; DOUR, H. J.; STANTON, A. L.; ROY-BYRNE, P. P.; STEIN, M. B.; et al. Anxiety and depressive symptoms and medical illness among adults with anxiety disorders. Journal of Psychosomatic Research, v. 78, n. 2, p. 109-115, 2015. Disponível em: <https://www.ncbi.nlm.nih.gov/pubmed/25510186>. doi: 10.1016/j.jpsychores.2014.11.018.

O'HAIRE, M. E.; GUÉRIN, N. A.; KIRKHAM, A. C. Animal-Assisted Intervention for trauma: a systematic literature review. Frontiers in psychology, v. 6, n. 1121, p. 113, 2015.2 Disponível em: < https://www.ncbi.nlm.nih.gov/pmc/articles/PMC4528099/>. doi: 10.3389/fpsyg.2015.01121

OUAKININ, S. R. S. Anxiety as a Risk Factor for Cardiovascular Diseases. Frontiers in psychiatry, v. 7, n. 25, p. 1-3, 2016. Disponível em: < https://www.ncbi.nlm.nih.gov/pmc/articles/PMC4764710/>. doi: 10.3389/fpsyt.2016.00025

PEPPE, A.; COStA, A.; Cerino, S.; CALTAGiRONE, C.; AlleVA, E.; et al. Targeting gait and life quality in persons with Parkinson's disease: Potential benefits of Equine-Assisted Interventions. Parkinsonism \& Related Disorders, v. 47, p. 9495, 2018. Disponível em: <https://www.ncbi.nlm.nih.gov/pubmed/29248341>. doi: 10.1016/j.parkreldis.2017.12.003

RAJE, K.; OJHA, S.; LOKESHA, E.; MUWEL, N.; CHOUDHARY, S.; et al. AnimalAssisted Therapy: Role of Animals as a Therapeutic. Research \& Reviews: Journal of Dairy Science and Technology, v. 7, n. 1, p. 18-21, 2018. Disponível em: < 
http://sciencejournals.stmjournals.in/index.php/RRJoDST/article/view/820>.

RASQUINHA, D. M.; ACHARYA, B. Relationship between Depression and Quality of Life among Institutionalised Elderly. International Journal of Scientific Research, v. $2, \quad$ n. $10, \quad$ p. 1-3, 2013. Disponível em: $<$ http://citeseerx.ist.psu.edu/viewdoc/download?doi=10.1.1.683.1714\&rep=rep1\&type $=$ pdf $>$.

ROBERTS-CLARKE, D.; FORNUSEK, C.; SAIGAL, N.; HALAKI, M.; BURNS, J.; et al. Relationship between physical performance and quality of life in Charcot-MarieTooth disease: a pilot study. Journal of the Peripheral Nervous System, v. 21, n. 4, p. 357-364, 2016. Disponível em: < https://www.ncbi.nlm.nih.gov/pubmed/27699915>. doi: 10.1111/jns.12191.

ROMANIUK, M.; EVANS, J.; KIDD, C. Evaluation of an equine-assisted therapy program for veterans who identify as 'wounded, injured or ill' and their partners. PLOS ONE, v. 13, n. 9, p. e0203943, 2018. Disponível em: <https://journals.plos.org/plosone/article?id=10.1371/journal.pone.0203943>. $\quad$ doi: 10.1371/journal.pone.0203943.

SINGEWALD, N.; SCHMUCKERMAIR, C.; WHITTLE, N.; HOLMES, A.; RESSLER, K. J. Pharmacology of cognitive enhancers for exposure-based therapy of fear, anxiety and trauma-related disorders. Pharmacology \& Therapeutics, v. 149, p. 150-190, 2015. Disponível em: <https://www.ncbi.nlm.nih.gov/pubmed/25550231>. doi: 10.1016/j.pharmthera.2014.12.004

SOUZA, J. M. G.; CASTELLI, G. M.; PAZ, L. P. S.; MORAES, A. G.; SILVA, M. L. Qualidade de Vida de cuidadores de praticantes de equoterapia no Distrito Federal. Saúde em Debate, v. 42, n. 118, p. 736-743, 2018. Disponível em: < http://www.scielo.br/scielo.php?script=sci_arttext\&pid=S0103-11042018000300736>. doi: 10.1590/0103-1104201811816.

SPIELBERGER, C. D.; GORSUCH, R. L.; LUSHENE, R. E. Manual for the StateTrait Anxiety Inventory. 1. ed. Palo Alto, CA: Consulting Psychologists Press, 1970.

STEIN, M. B.; CRASKE, M. G. Treating Anxiety in 2017: Optimizing Care to Improve Outcomes. JAMA, v. 318, n. 3, p. 235, 2017. Disponível em: < https://www.ncbi.nlm.nih.gov/pubmed/28679009>. doi: 10.1001/jama.2017.6996.

STEIN, M. B.; SAREEN, J. Generalized Anxiety Disorder. New England Journal of Medicine, v. 373, n. 21, p. 2059-2068, 2015. Disponível em: < https://www.ncbi.nlm.nih.gov/pubmed/26580998>. doi: 10.1056/NEJMcp1502514

STERGIOU, A.; TZOUFI, M.; NTZANI, E.; VARVAROUSIS, D.; BERIS, A.; et al. Therapeutic Effects of Horseback Riding Interventions: A Systematic Review and Meta-analysis. American journal of physical medicine \& rehabilitation, v. 96, n. 10, p. 717-725, 2017. Disponível em: < https://www.ncbi.nlm.nih.gov/pubmed/28252520>. doi: 10.1097/PHM.0000000000000726. 
STERN, C.; CHUR-HANSEN, A. An umbrella review of the evidence for equineassisted interventions. Australian Journal of Psychology, v. 2019, n. Special Issue, p. 1-14, 2019. Disponível em: < https://aps.onlinelibrary.wiley.com/doi/10.1111/ajpy.12246>. doi: 10.1111/ajpy.12246

THE WHOQOL GROUP. Development of the World Health Organization WHOQOLBREF Quality of Life Assessment. Psychological Medicine, v. 28, n. 3, p. 551-558, 1998b. Disponível em: < https://www.ncbi.nlm.nih.gov/pubmed/9626712>.

THE WHOQOL GROUP. The World Health Organization Quality of Life Assessment (WHOQOL): development and general psychometric properties. Social science \& medicine (1982), v. 46, n. 12, p. 1569-85, 1998a. Disponível em: <https://www.sciencedirect.com/science/article/abs/pii/S0277953698000094?via\%3D ihub\#!>. doi: 10.1016/S0277-9536(98)00009-4

WAITE, T. C.; HAMILTON, L.; O'BRIEN, W. A meta-analysis of Animal Assisted Interventions targeting pain, anxiety and distress in medical settings. Complementary Therapies in Clinical Practice, v. 33, p. 49-55, 2018. Disponível em: $\quad$ <https://www.ncbi.nlm.nih.gov/pubmed/30396626>. doi: 10.1016/j.ctcp.2018.07.006. 